\title{
Polygonal finite elements for finite elasticity
}

\author{
Chi, Heng, hengchi2@illinois.edu; Talischi, Cameron; Lopez-Pamies, Oscar; Paulino, Glaucio, U of I at \\ Urbana-Champaign, United States
}

\begin{abstract}
Nonlinear elastic materials are of great engineering interest, but challenging to model with standard finite elements. The challenges arise because nonlinear elastic materials are characterized by nonconvex stored-energy functions as a result of their ability to undergo large reversible deformations, are incompressible or nearly incompressible, and often times possess complex microstructures. In this study, we propose and explore an alternative approach to model finite elasticity problems in two dimensions by using polygonal discretizations. We present both lower order displacement-based and mixed polygonal finite element approximations, the latter of which consist of a piecewise constant pressure field and a linearly-complete displacement field at the element level. Through numerical studies, the mixed polygonal finite elements are shown to be stable and convergent. For demonstration purposes, we deploy the proposed polygonal discretization to study the nonlinear elastic response of rubber filled with random and periodic distributions of rigid particles, as well as the development of cavitation instabilities in elastomers containing vacuous defects. These physically based examples illustrate the potential of polygonal finite elements in studying and modeling nonlinear elastic materials with complex microstructures under finite deformations.
\end{abstract}

